\section{Cahiers de Narratologie}

Analyse et théorie narratives

10.2 | 2001

La voix narrative

\title{
Les Os de seiche entre roman poétique du moi et altérité
}

\section{Agostino Salpetrio}

\section{(2) OpenEdition}

1 Journals

Édition électronique

URL : http://journals.openedition.org/narratologie/10287

DOI : 10.4000/narratologie.10287

ISSN : 1765-307X

Éditeur

LIRCES

\section{Édition imprimée}

Date de publication : 1 janvier 2001

Pagination : 415-424

ISBN : 2914561032

ISSN : $0993-8516$

\section{Référence électronique}

Agostino Salpetrio, «Les Os de seiche entre roman poétique du moi et altérité », Cahiers de Narratologie [En ligne], 10.2 | 2001, mis en ligne le 01 janvier 2001, consulté le 11 juin 2020. URL : http://

journals.openedition.org/narratologie/10287 ; DOI : https://doi.org/10.4000/narratologie.10287 


\title{
LES OS DE SEICHE ENTRE ROMAN POÉTIQUE DU MOI ET ALTÉRITÉ
}

\author{
Agostino SALPIETRO \\ Université de Nice-Sophia Antipolis
}

Affronter la question du rapport à l'Autre dans la série Mediterraneo, (Flot méditerranéen), des Os de Seiche d'Eugenio Montale amène inévitablement à parcourir des sentiers qui sembleraient à première vue nous éloigner de l'exercice traditionnel de la critique littéraire, mais il n'en va pas ainsi. Au contraire, l'expérience poétique montalienne s'ouvre dans sa dimension plus authentique justement dans ce double détournement, dans cet apparent hors-piste. Une fois empruntées ces " viuzze che seguono i ciglioni »', comme le dit le poète, qui semblent nous conduire dans des lieux étranges ou peu fréquentés par les critiques, voilà qu'on se retrouve tout d'un coup au centre des questions fondamentales du langage et de la poésie.

Mediterraneo (Flot méditerranéen) pourrait être défini comme un roman poétique de l'Altérité. Mais cette définition mérite d'être expliquée préliminairement et circonstanciée malgré son évidence apparente.

Nous voudrions montrer comment dans Mediterraneo, le sujet-poète, dans sa relation économique avec l'Altérité et ses diverses formes, plus que se replier lyriquement sur lui-même, tend au contraire à un double mouvement : un mouvement qu'on pourrait définir d'enthousiasme (dans la définition platonicienne d'un Dieu à l'intérieur de $\mathrm{soi}^{2}$ ), et l'autre que nous définirons comme extase (la sortie du Moi de sa conscience de soi).

${ }^{1}$ Eugenio MONTALE, L'opera in versi, Edizione critica a cura di R. Bettarini e G. Contini, Einaudi, Torino 1980.

${ }^{2}$ Cf. PlatonE, Ione, 538 ; Plotino Enneadi, VI, 9, 11, 13. 
La constitution de cette topologie et ontologie de l'altérité sera de toute façon poétique. La parole, bien que soit claire son intention définitoire et spéculative, ne perd pas sa spécificité poétique (même si nous sommes dans une zone de la poésie de Montale que la critique a identifiée comme l'une des plus prosaïque ${ }^{3}$ ), mais au contraire la dialectique poésie/non poésie se charge de ramener la parole à son dire originaire, à un cosmos dans lequel, au delà du rapport mimétique entre monde et langage, s'instaure une consonance significative, un isomorphisme, entre parole et nature, entre Moi et non-Moi. Ce n'est pas par hasard que le texte Potessi almeno costringere, qui est une déclaration de poétique, se place en avant-dernière position de la série. On a donc dit roman, mais également dialogue, colloque toujours proche de l'annulation de l'un ou l'autre agoniste. Mais quelle est le sens du dialogue qui s'ouvre dans Mediterraneo ? Sommes-nous dans la situation d'un dialogue comme acte d'amour pour l'Autre (ou de l'Autre), ou dans celle du dialogue comme irruption du tragique dans les vicissitudes humaines, comme reconnaissance du danger de la parole qui rencontre l'Autre que soi ?

Nous adopterons donc deux critères, deux références, afin de vérifier comment Mediterraneo se place sur cette double dimension de l'écriture poétique. Le premier critère concerne la question de la position absolue ${ }^{4}$ dans les $O s$ de seiche en général. Le second naîtra quant à lui d'une relecture de cette présence éthique que la critique ${ }^{5}$ a souvent reconnu dans Mediterraneo.

Que signifie le fait de se poser le problème de la position absolue dans les $O s$ de seiche ? Analysons le vers de Vento $e$ bandiere (Vent et bannières) : "Il mondo esiste... Uno

3 Cf. Gianfranco CONTINI, Una lunga fedeltà, Einaudi, Torino 1974, pag. $24:$ : [...] la serie Mediterraneo è da considerarsi come una prosa riflessiva intorno al simbolo stesso dell'indifferenza, ordine o legge di varietà-fissistà, il mare $[\ldots]$ ».

${ }^{4}$ Cf. Martin HEIDEGGER, Die Grundprobleme der Phänomenologie, Vittorio Klostermann Verlag, 1975.

5 Cf. Marco FORTI, Eugenio Montale. La poesia, la prosa di fantasia e d'invenzione, Mursia, Milano 1973-1983, pagg. 90 e ss. ; Romano Luperini, Storia di Montale, Laterza, Bari 1992, pagg. 36 e ss. 
stupore arresta | il cuore [...]» (Le monde existe... Une stupeur arrête | le cœur $\left.[\ldots]^{6}\right)$. Qu'est ce que le "monde » dans les $O s$ ? Le définir comme un horizon unitaire, à l'intérieur d'un système poétique qui s'ordonne de façon binaire comme celui des $O s$ devient problématique. La parole " monde » nous ouvre cependant à une compréhension plus profonde de la poétique montalienne. S'il est vrai que la poésie de Montale pose radicalement la question de la connaissance, problématique, de la réalité7, alors le "monde » devra être cet horizon transcendantal qui contient l'ordre dans lequel les objets peuvent ou ne peuvent pas se donner à l'intentionnalité de l'être-là historique du poète-sujet. C'est pour cette raison que chez Montale l'Altérité elle-même devra de quelque façon s'ordonner de façon binaire : l'autre que soi en tant qu'objet, l'Autre que soi en tant que sujet capable lui aussi d'intentionnalité. Le monde est la substance de l'objet (la non-existence ${ }^{8}$ ) dans l'ici et maintenant, l'hic et nunc, qui entre en rapport d'intentionnalité avec un sujet qui est déjà en dehors de soi, s'autotranscendant, qui séjourne déjà de façon problématique auprès de l'objet.

«Le monde existe » signifie chez Montale l'affirmation d'une complexité qui mérite d'être traversée tout en en postulant l'être absolu, la radicale immanence qui appelle le sujet hors de lui et le pose auprès de l'objet qui inaugure ce monde. C'est ici que le discours montalien se révèle comme étant phénoménologique : «... Uno stupore arresta | il cuore » (Une stupeur arrête I le cœur). C'est l'établissement d'une suspension, d'une époché qui, si elle suspend d'une part l'activité descriptive et ordonnatrice du sujet par rapport au monde, permet par ailleurs à son intentionnalité de le conduire auprès de l'objet-monde de sorte que cette pré-compréhen-

6 Eugenio MONTALE, Poésies, traduction de Patrice Angelini, Gallimard, Paris 1966.

${ }^{7}$ Cf. Michel CASSAC, Ossi di seppia ou le recit du mythe identitaire (Revue des Études Italiennes, t. XLIV, 3-4/1998) : "Dans Ossi di Seppia, par images instantanées et discontinues, la mémoire en lambeaux tente de comprendre, en saisissant l'apparence d'une réalité dont il ne reste que la forme de l'incompréhension ».

8 Cf. Gianfranco CONTINI, Una lunga fedeltà, Einaudi, Torino 1974, pagg. 19-ss. 
sion qu'il est ne lui empêche pas de saisir la chose, l'objet dans sa double présence, en tant que placée et décrite par le sujet et en tant qu'origine du rapport, de l'historicité du sujet. C'est ainsi que la lecture de Mediterraneo ne saurait se passer de la constatation de cette double tension qui investit l'objet, surtout afin d'éviter une lecture qui, au lieu de nous guider vers le statut fondamental du rapport à l'objet, aboutisse à une interprétation vitaliste et irrationnelle.

On procédera maintenant à une lecture critique des neuf mouvements dans lesquels s'articule Mediterraneo afin d'y voir d'un côté le déploiement d'une structure-roman thématique qui ne couvre pas le vers mais, au contraire, qui l'accompagne, et de l'autre cette manifestation du rapport à l'Altérité qui est le sujet de cette intervention.

La poésie qui ouvre la suite, $A$ vortice s'abbatte prépare pour ainsi dire la scène avant la représentation. C'est un moment de préparation à la rencontre avec l'Altérité qui trouve sa représentation dans la figure de la mer, mer qui est entrevue de loin et pas encore définie dans la clarté d'un dialogue ouvert : « e al mare là in fondo fa velo I più che i rami, allo sguardo, l'afa che a tratti erompe I dal suolo che si avvena ». Toute la première partie de la poésie est dominée par des figures qui indiquent une descente, une chute : «s'abbatte », « terra percorsa da sghembe I ombre di pinastri », "s'ingorgano", "ripiovere I di schiume sulle rocce». Pourtant ce n'est que dans la partie finale qu'explose, inattendu et incommensurable par rapport aux prémisses, le miracle : le vol vertical, soudain, de deux geais. C'est seulement ici que la poésie s'ouvre à la rencontre de la mer. Avec ce vol fulgurant, avec ce visage redressé, il est donné au poète de suspendre la dimension temporelle qui le lie à la détermination chronologique et de se poser dans l'instant qui s'ouvre, à l'écoute de la voix qui vient du lointain absolu.

Après ce premier moment de verticalité, Montale commence son difficile dialogue avec la mer, dialogue qui le forcera à redéfinir le statut de la réalité et du sujet dans sa totalité. C'est le moment de Antico, sono ubriacato dalla voce (Ancien, je suis enivré de la voix) où se concentre la réflexion poétique sur la loi, sur l'objet, sur l'être seulement que créature. Pour ce qui est de l'objet, ce qui nous occupe 
ici, il sera intéressant de voir comment celui-ci est mis immédiatement en relation avec l'abîme, avec la profondeur mystérieuse de la mer, de l'Altérité : "sbatti sulle sponde I tra sugheri alghe asterie | le inutili macerie del tuo abisso » (rabats sur les rives I parmi bouchons algues étoiles de mer I les inutiles décombres de ton abîme). Les objets deviennent « inutili macerie » (les inutiles décombres), la définition de l'objet est ainsi privée d'une quelconque fondation physicométaphysique et la signification est ramenée à des catégories existentielles et non plus rationnelles ou cognitives'. L'objet est ainsi transcendé dans sa simple subsistance justement grâce à sa provenance des profondeurs de la mer, tandis que le sujet lui aussi en vient à se trouver dans une condition transcendante à la fois grâce au sentiment d'être créature qui s'instaure entre le poète et la mer ( « tu m'hai detto primo I che il piccino fermento I del moi cuore non era che un momento I del tuo ", Tu m'as dit le premier I que le frêle effervescence I de mon cœur n'était qu'un moment I de la tienne), mais également à cause de ce «come tu fai » (comme toi) qui rapproche le sujet de la dimension de l'autre-que-soi. Voici donc que sont définis trois degrés : l'être absolu de la mer, l'être-là du poète en relation économique avec l'Altérité et la subsistance des objets qui sont réduits à « inutili macerie » (les inutiles décombres).

Dans le troisième mouvement, Scendendo qualche volta (En descendant quelquefois), Montale affronte les questions de la modalité de cet impossible dialogue. On arrive ici à un moment caractéristique de la poésie montalienne, celui de la suspension de la temporalité comme chronologie : «non m'era più in cuore la ruota I delle stagioni e il gocciare I del tempo inesorabile » (je n'avais plus dans le cœur la roue I des saisons et le goutte à goutte I du temps inexorable). Ce vers établit deux aspects de la temporalité inauthentique: la roue représente le cycle éternel qui n'a pas la liberté de changer l'identique qu'il représente et qui agit donc comme protection contre le devenir, si nous pensons à l'archétype jungien du

9 Cf. Gianfranco CONTINI, Una lunga fedeltà, Einaudi, Torino 1974, pag. 82: " Il primum di Montale sta molto più addietro, è in un minimo di tollerabilità al vivere ». 
cercle $^{10}$, mais qui agit également comme obstacle à l'évènement, au miracle, au fait inattendu qui défie la loi naturelle et devient miracle libératoire ; l'inexorabilité concerne quant à elle le fait que la dimension chronologique du temps ne connaisse ni rachat ni pietas pour l'homme. Sans oublier qu'ici le «cœur » n'est pas le lieu du sentiment mais plutôt celui de l'appréhension du sentiment du temps.

Dans le quatrième mouvement nous voyons comment l'objet s'enroule dans une réverbération existentielle salvateur : " mi condanna I s'io lo tento anche un ciotolo I ròso sul moi cammino, I impietrato soffrire senza nome, I o l'informe rottame | che gittò fuor del corso la fiumara | del vivere in un fitto di ramure e di strame » (je suis condamné I si je le tente, même par un galet I usé sur mon chemin, I souffrance anonyme de pierre, ou par l'épave informe I qu'a rejetée de son cours le torrent I de la vie en un fouillis de branchages et de ronces). La « substance» de l'objet est bouleversée et celui-ci en vient à assumer une valeur existentielle justement grâce à cette provenance autre par laquelle il est touché et privé de forme. L'objet devient alors la position intermédiaire qui dans la narration se pose entre le poète et l'Altérité. C'est ainsi que la question montalienne de l'Autre dans Mediterraneo passe inévitablement par une redéfinition du statut objectal, qui se révèle ici comme le point focal où coexistent deux formes d'intentionnalité : celle du Moi du poète, qui dans son séjour auprès de l'objet sent sa condamnation, et celle de l'Altérité qui rend l'objet significatif, mais non pas d'une signification claire et évidente, mais plutôt d'une signification liée au mystère de l'Altérité comme Indistinct. De l'Indistinct on passe alors à une forme en mineur, faible, d'indistinction : le « senza nome » (anonyme).

Il y a ensuite le moment où Montale s'attache à éviter une lecture trop anthropomorphe de l'Autre. C'est la cinquième partie de Mediterraneo, son moment central : Giunge a volte repente (Parfois, soudaine, arrive). Le "cuore disumano » (ton cœur inhumain) indique l'éloigne-

${ }^{10}$ Cf. Maryse Jeuland MEYNAUD, Oggetti e archetipi nella poesia di E. Montale. Dagli Ossi di seppia alla Bufera (AA. VV., La poesia di Eugenio Montale. Atti del convegno Internazionale tenuto a Genova dal 25 al 28 novembre 1982, Le Monnier, Firenze 1984), pagg. 21-ss. 
ment profond par rapport à l'Autre, lorsqu'on le scrute non pas dans un rapport économique avec le Moi poétant (en tant qu'objet-signal) mais dans son être-en-soi, dans son essence intime, libre et abyssale. Le poète dit en effet : " In me ripiego, vuoto I di forze, la tua voce pare sorda » (Je me replie en moi, vide I de forces, ta voix paraît sourde). C'est là l'impossibilité du sujet à se transcender afin de séjourner auprès de l'objet significatif, du moment que la concentration en soi de l'Autre la restitué à sa simple substance. " M'affisso nel pietrisco I che verso te digrada » (Je fixe la pierraille | qui descend vers toi par degrés) a le sens d'un retour à un statut de subsistance. Il n'y a plus de « ciottolo I ròso [...] I impietrato soffrire senza nome » (un galet I usé [...] | souffrance anonyme de pierre), mais un "pietrisco » (pierraille) qui est un viatique vers le mystère, un pur signal vide de sens qui ne soit pas celui de l'être-entre.

Dans le sixième mouvement Montale définit une sorte d'éthique de la création poétique, de laquelle ressortent quelques idées fondamentales: la poésie accueille (peut accueillir) une partie du « don » que l'Altérité fait à l'homme : ce don est la mémoire immémorable de l'autre comme origine $\mathrm{du}$ sujet. C'est in nuce, et une fois faites les nécessaires distinctions, quelque chose de très proche du colloque hölderlinien" ", où semblent nous conduire également les derniers vers du poème « $\mathrm{E}$ un giorno queste parole senza rumore I che teco educammo nutrite I di stanchezze e di silenzi, I parranno a un fraterno cuore I sapide di sale greco » (Et un jour ces paroles muettes I qu'avec toi nous avions nourries I de lassitudes et de silences, sembleront à un cœur fraternel I toutes savoureuses de sel grec). Le poème contient donc un aspect éthique, un "devoir être » mais qui est en réalité une indication ontologique fondatrice : la nature intime de la poésie comme colloque qui naît de l'écoute de la voix de l'Autre.

Dans la partie suivante, Avrei voluto sentirmi scabro ed essenziale (J'aurais voulu me sentir épuré, essentiel), s'ouvre une nouvelle définition de l'objet au sein de la

11 Cf. Martin HeIDEGGER, Hölderlin e l'essenza della poesia, Conferenza tenuta all'Istituto Italiano di Studi Germanici il 2 aprile 1936. 
dynamique binaire que nous avons indiquée : "Avrei voluto sentirmi scabro ed essenziale I siccome i ciottoli che tu volvi, I mangiati dalla salsedine ; | scheggia fuor del tempo, testimone | di una volontà fredda che non passa. I Altro fui : uomo intento che riguarda I in sé, in altrui, il bollore I della vita fugace uomo che tarda I all'atto, che nessuno, poi, distrugge " (J'aurais voulu me sentir épuré, essentiel, I comme les galets que tu roules, I mangés par le sel ; I éclat hors du temps, témoin I d'une volonté froide qui ne passe pas. I Je fus autre : homme attentif qui regarde I en lui-même, en autrui, l'effervescence I de la vie fugace - homme qui tarde I à l'acte, quel nul, ensuite, ne détruit). Que signifie cette impossible tentative de renoncer à sa propre humanité en faveur de l'inorganique sinon la faillite d'une intentionnalité injustement interprétée comme "volonté de renoncement»? C'est la question du mal, qui dans le cercle Sujet-Altérité se pose comme l'impossibilité du vouloir humain de définir ce qui ne peut être exprimé par le langage. Lorsqu'on cherche le sens du « il male che tarla il mondo » (le mal | qui ronge le monde) la réalité dans son ensemble perd toute possibilité de signification authentique. Il y a là l'effondrement radical d'un statut cognitif qui tente un pas au-delà de la dimension limite de l'homme.

On arrive ainsi à l'avant-dernier passage de cet enchâ̂nement de textes. Le lecteur se trouve face à un second moment de déclaration poétique, nouveau, qui ajoute, par rapport au premier, la positivité du désir, et révèle le sens ultime de notre recherche : l'abandon. Laissant de côté, maintenant, la question du poétique, voyons comment, dans la définition de l'abandon, se concentre le vrai discours onthologico-cognitif du rapport Sujet-Altérité. Si l'on pouvait croire jusque-là que le cadre phénoménologique de Montale s'arrêtait à une epoché qui se résout en une prédominance de la sensation sur la rationalité, voici maintenant que le poète explique clairement que cela n'est nullement le cas. L'epoché est celle de la pensée, mais également celle des sens et du sens. C'est le reflux de l'illimité qui est la " vastità », la mer, l'Etre. L'Autre devient expérience possible à travers le poétique, qui est ici suspension totale de soi jusqu'à l'extase. Le sujet est totalement hors-de-soi, et, en dehors du monde de la simple subsistance, il reconquiert une dernière possibilité 
cognitive au prix d'une perte de l'identité reconnaissable, du soi : « M'abbandonano a prova i miei pensieri. I Sensi non ho ; né senso. Non ho limite».

Mediterraneo se conclut enfin avec un texte où ce rapport entre Altérité et sujet que nous avons suivi jusqu'ici révèle son sens ultime et fondamental : «Bene lo so : bruciare, | questo, non altro, è il mio significato » (Oui je le sais : brûler, c'est cela, et rien d'autre, mon sens.). C'est ici que nous est offerte la possibilité de comprendre ce plus de sens qui n'était pas donné, pas posé initialement. Cet ajout de sens, c'est l'acceptation du destin, c'est le souvenir de l'immémorable (« sbigottimento mi prese I quale d'uno scemato di memoria I quando si risovviene del suo paese » un trouble m'a saisi I comme l'infirme de mémoire I lorsqu'il se ressouvient de son pays). Cette leçon apprise par le sujet est une véritable révélation existentielle : la caducité de l'homme est le miroir du « esser vasto e diverso I e insieme fisso » (être vaste e divers I et en même temps fixe) de l'Être, qui trouve dans l'image de la mer une possibilité d'expression dans le langage.

Voilà donc que nous pouvons conclure cette intervention en citant un texte qui, encore une fois, nous éloigne en apparence seulement de la critique littéraire, parce qu'il définit avec rigueur la possibilité d'identifier dans ce poème-roman qu'est Mediterraneo cette dimension de l'abandon qui permet à l'homme de rencontrer l'objet et l'Altérité dans leur dimension authentique : " $\mathrm{A}$ travers la contenance de la Gelassenheit (l'abandon) nous pouvons ouvrir les portes à l'interrogation pour quelque chose que nous ne precomprenons pas par notre savoir. Dans cet espace humble et limité en attendant sans ad-tendere, sans adtesa qui prétende devancer l'attendu, [...], une simple contenance recueillie, nous pouvons nous ouvrir sur le mystère de l'autre, tout en sachant que l'autre ne pourrait jamais nous atteindre : nous pourrons même l'attendre pendant des millénaires sans que notre attente vide en favorise la venue. Mais ce c'est que dans cette contenance qui approfondi, sur la ligne du vide, dans le désert du tout qui se transforme en rien, [...], que nous pouvons écouter l'éventuel se donner gratuit d'un mot 
imprévisible et incostruibile, capable d'ouvrir à nouveau l'histoire au se donner libre de la vérité de l'être ${ }^{12}$.

Voilà ce qu'est Mediterraneo, cette "contenance de l'abandon " qui non seulement se présente comme une exceptionnelle possibilité de connaître, mais également comme moteur et modalité d'un rapport qui redéfini et réécrit, poétiquement, le rapport avec les objets et l'Autre que soi.

12 Francesco TOMATIS, Escatologia della negazione, Città Nuova, Roma 1999, pag. 55. 